PREPARED FOR THE U.S. DEPARTMENT OF ENERGY, UNDER CONTRACT DE-AC02-76CH03073

PPPL-3744

PPPL-3744

UC-70

Simulating Photons and Plasmons

in a Three-dimensional Lattice

by

A. Pletzer and G. Shvets

September 2002

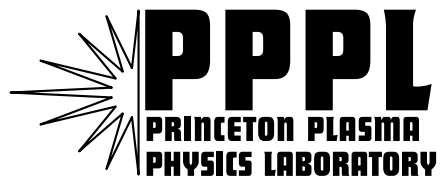

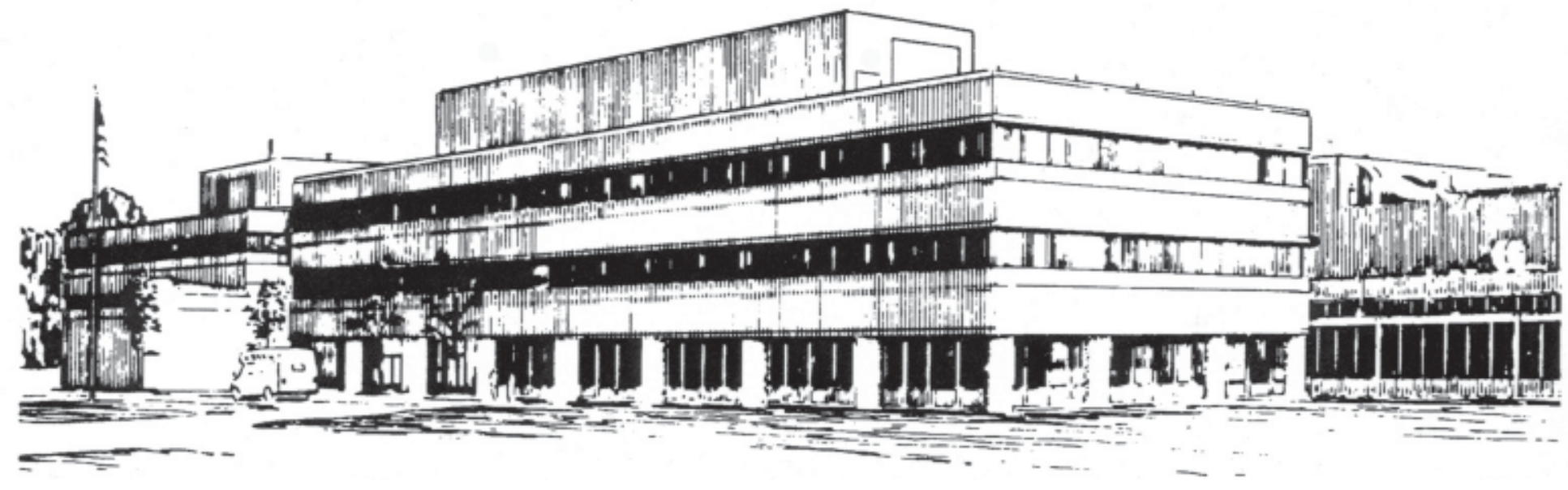

PRINCETON PLASMA PHYSICS LABORATORY PRINCETON UNIVERSITY, PRINCETON, NEW JERSEY 


\section{PPPL Reports Disclaimer}

This report was prepared as an account of work sponsored by an agency of the United States Government. Neither the United States Government nor any agency thereof, nor any of their employees, makes any warranty, express or implied, or assumes any legal liability or responsibility for the accuracy, completeness, or usefulness of any information, apparatus, product, or process disclosed, or represents that its use would not infringe privately owned rights. Reference herein to any specific commercial product, process, or service by trade name, trademark, manufacturer, or otherwise, does not necessarily constitute or imply its endorsement, recommendation, or favoring by the United States Government or any agency thereof. The views and opinions of authors expressed herein do not necessarily state or reflect those of the United States Government or any agency thereof.

\section{Availability}

This report is posted on the U.S. Department of Energy's Princeton Plasma Physics Laboratory Publications and Reports web site in Fiscal Year 2002. The home page for PPPL Reports and Publications is: http://www.pppl.gov/pub_report/

DOE and DOE Contractors can obtain copies of this report from:

U.S. Department of Energy

Office of Scientific and Technical Information

DOE Technical Information Services (DTIS)

P.O. Box 62

Oak Ridge, TN 37831

Telephone: (865) 576-8401

Fax: (865) 576-5728

Email: reports@adonis.osti.gov

This report is available to the general public from:

National Technical Information Service

U.S. Department of Commerce

5285 Port Royal Road

Springfield, VA 22161

Telephone: 1-800-553-6847 or

(703) 605-6000

Fax: (703) 321-8547

Internet: http://www.ntis.gov/ordering.htm 


\title{
Simulating photons and plasmons in a three-dimensional lattice
}

\author{
A. Pletzer ${ }^{a}$, and G. Shvets ${ }^{b}$ \\ a Princeton Plasma Physics Laboratory \\ PO Box 451 \\ Princeton NJ 08543 \\ b Illinois Institute of Technology \\ 3101 South Dearborn \\ Chicago, IL 60616
}

\begin{abstract}
Three-dimensional metallic photonic structures are studied using a newly developed mixed finite element-finite difference (FE-FD) code, Curly3d. The code solves the vector Helmholtz equation as an eigenvalue problem in the unit cell of a triply periodic lattice composed of conductors and/or dielectrics. The mixed FE-FD discretization scheme ensures rapid numerical convergence of the eigenvalue and allows the code to run at low resolution. Plasmon and photonic band structure calculations are presented.
\end{abstract}

Key words: Band structure, Helmholtz equation, 3-D, mixed FE-FD, perfect conductor

\section{Background}

The recent upsurge of interest in the electrodynamics of three-dimensional periodic structures is due to the realization that composite materials can exhibit unusual electromagnetic properties. For example, a composite material made of poles and split ring-resonators can display negative dielectric permittivity and magnetic permeability for a range of frequencies [1]. Such materials known

1 This work supported by the U.S. Department of Energy under DE-AC02$76 \mathrm{CH} 03073$. 
as left-handed materials support electromagnetic waves with a very counterintuitive behavior: their group and phase velocities oppose each other. Lefthanded materials can be used for developing, for example, "perfect" lenses [2] capable of sub-wavelength resolution.

Key to understanding the bulk properties of the infinitely extended composite materials is the accurate determination of the band structure of the electromagnetic waves by solving Maxwell's equations. In the frequency domain, the wave equation becomes

$$
\boldsymbol{\nabla} \times\left(\mu^{-1} \cdot \nabla \times \mathbf{E}\right)=\frac{\omega^{2}}{c^{2}} \epsilon \cdot \mathbf{E}
$$

where $\mathbf{E}$ is the electric field and $\mu(\epsilon)$ the permeability (permittivity) of the medium. Both $\epsilon$ and $\mu$ are spatially varying tensors. The perfect conductor boundary condition $\mathbf{E} \times \mathbf{n}=0$, where $\mathbf{n}$ is the normal, is applied at the conductor boundaries. Because of the system periodicity, and according to Floquet's theorem, the electric field can be expressed as $\tilde{\mathbf{E}} \operatorname{expik} \cdot \mathbf{x}$, where $\tilde{\mathbf{E}}(\mathbf{x}+\mathbf{L})=\tilde{\mathbf{E}}(\mathbf{x})$ is a periodic function, $\mathbf{L}$ is any one of the periodicity vectors, and $\mathbf{k}$ is inside the Brillouin zone. Therefore, it is sufficient to solve Eq. (1) inside the unit cell, which we assume to be a parallelepiped with the dimensions $L_{x} \times L_{y} \times L_{z}$. The equation for $\tilde{\mathbf{E}}$ can be obtained from (1) by replacing $\boldsymbol{\nabla}$ by $\nabla+\mathrm{ik}:$

$$
(\boldsymbol{\nabla}+\mathrm{ik}) \times\left[\mu^{-1} \cdot(\boldsymbol{\nabla}+\mathrm{ik}) \times \tilde{\mathbf{E}}\right]=\frac{\omega^{2}}{c^{2}} \epsilon \cdot \tilde{\mathbf{E}}
$$

Here, $\mathbf{k} \cdot \mathbf{L}$ represents, up to a term $2 \pi(m+n+l)(m, n$ and $l$ are integers), the phase shift experienced by the wave across the unit cell. For $m=n=l=0$, $\mathbf{k}$ can be thought of as the wave vector of an incident wave. From now on we will focus on $\tilde{\mathbf{E}}$ and drop the $\sim$ for notational simplicity.

Note that we have chosen here to write the equation for $\mathbf{E}$. The equation for the magnetic field $\mathbf{H}$ is similar to (2) except for $\epsilon$ and $\mu$ playing opposite roles. Hence, the pairs $(\epsilon, \mathbf{E})$ and $\left(\mu^{\dagger}, \mathbf{H}^{*}\right)$ can be regarded as dual of each other (the complex $^{*}$ and Hermitian ${ }^{\dagger}$ conjugates are required to derive the Poynting flux involving $\mathbf{E}$ only from an expression solely based on $\mathbf{H}$ and vice-versa). This duality extends to the jump conditions across permittivity discontinuities $([[\mathbf{n} \cdot \epsilon \cdot \mathbf{E}]]=0)$ and permeability discontinuities $([[\mathbf{n} \cdot \mu \cdot \mathbf{H}]]=0)$ but not, however, to the boundary conditions at the surface of perfect conductors. While the tangential $\mathbf{E}\left(\mathbf{E}_{t}\right)$ must be set to zero there, it is the normal component of $\mathbf{H}\left(H_{n}\right)$ that is required to vanish at the conductor so that the mode structure of magnetic and electric fields will in general to be fundamentally different. It turns out that solving for $\mathbf{H}$ is from a coding viewpoint straightforward because $H_{n}=0$ are the default (homogeneous natural) boundary conditions of 
the FE method. On the other hand, solving for $\mathbf{E}$ involves applying (explicit) Dirichlet conditions, which turns out to be numerically more efficient.

\section{Discretization}

In order to solve (2), the field (e.g. electric) is expanded $\mathbf{E}=\sum_{j} E_{j} \mathbf{e}_{j}(\mathbf{x})$ in finite vector elements $\mathbf{e}_{j}$. Our choice of $\mathbf{e}_{j}$ 's is motivated by the desire to capture accurately discontinuities at conductor and dielectric interfaces. Van Welij elements [3], by being piecewise linear in the directions perpendicular to the field but only piecewise continuous along the field, are well suited for this purpose. Such a spatial dependence ensures that each $\mathbf{e}_{j}$ is divergence-free within an elemental mesh unit. Van Welij elements do not appear to suffer from numerical pollution (spurious modes) [4], a problem that commonly afflicts other types of discretizations. However, van Welij elements require a rectilinear mesh. Although later generalized to curvilinear meshes [5], we found that using straight hexahedral (brick) elements has a number of advantages not least that the domain can be built incrementally. Each brick can be identified by a triplet $(i, j, k), i=1, \cdots N_{x}, j=1, \cdots N_{y}$ and $k=1, \cdots N_{z}$. Complex geometries can thus be constructed by adding and removing bricks at will, while noting that doing so has the disadvantage of introducing "staircase" interfaces.

The discretized version of the vector Helmholtz equation is obtained after multiplying (2) by a test function $\mathbf{e}_{i}$, integrating over the unit cell domain and applying the divergence theorem to yield the generalized eigenvalue system

$$
\sum_{j} A_{i j} E_{j}=\lambda \sum_{j} B_{i j} E_{j}
$$

where the eigenvalue is $\lambda \equiv \omega^{2} / c^{2}$,

$$
A_{i j}=\int d V(\boldsymbol{\nabla}+\mathrm{i} \mathbf{k})^{*} \times \mathbf{e}_{i} \cdot \mu^{-1} \cdot(\boldsymbol{\nabla}+\mathrm{ik}) \times \mathbf{e}_{j}
$$

and

$$
B_{i j}=\int d V \mathbf{e}_{i} \cdot \epsilon \cdot \mathbf{e}_{j}
$$

are two sparse matrices and integration is over the volume of the unit cell. To be exact, the integration by parts introduces a surface term $\oint d \boldsymbol{\sigma} \cdot \mathbf{e}_{i} \times\left[\mu^{-1} \cdot(\boldsymbol{\nabla}+\right.$ $\mathrm{ik}) \times \mathbf{E}]$ in the left-hand side of (3). But by virtue of $\mathbf{E}$ being periodic this term does not contribute on unit cell faces. Nor does it contribute on perfect con-

ductor faces since $\mathbf{E}_{t}=0$ there. Finally, it is straightforward to see that this 
term also cancels at conductor faces when solving for $\mathbf{H}$ (replace $\mu \rightarrow \epsilon$ and $\mathbf{E} \rightarrow \mathbf{H}$ in the above $)$ since we have $\left(\epsilon^{-1} \cdot[\boldsymbol{\nabla}+\mathrm{ik}] \times \mathbf{H}\right)_{t} \sim \mathbf{E}_{t}=0$.

So far we have not deviated from a standard implementation of the FE method. Integrals (4) and and (5) can be computed analytically assuming $\epsilon$ and $\mu$ to be constant in each brick, with the matrix $A$ yielding a stencil approximation of operator $(\boldsymbol{\nabla}+\mathrm{ik}) \times \mu^{-1} \cdot(\boldsymbol{\nabla}+\mathrm{ik}) \times$ and matrix $B$ an approximation to $\epsilon$. When $\epsilon=1$, the $B$-stencil for parallel edges

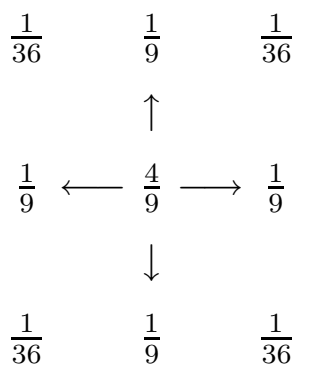

can be regarded as a crude approximation of the identity operator. Had we chosen an FD approach, then the coefficient $\frac{4}{9}$ would have been 1 with all other coefficients set to zero. This suggests that a certain degree of freedom is available in the choice of $B$-stencil. It can be shown that this choice in $B$-stencil does not compromise the uniqueness of the solution. Rather, it reflects the fact that the numerical error is dominated in the FE method by the $A$-stencil at high resolution with modifications of $B$ allowed provided they are of same or higher order of accuracy. This degree of freedom is therefore similar to the choice of quadrature method in the tunable integration scheme of Bondeson and $\mathrm{Fu}[6]$, and the motivation in Curly3d for introducing a free parameter $\Delta$, which allows for some flexibility in the choice of B-stencil

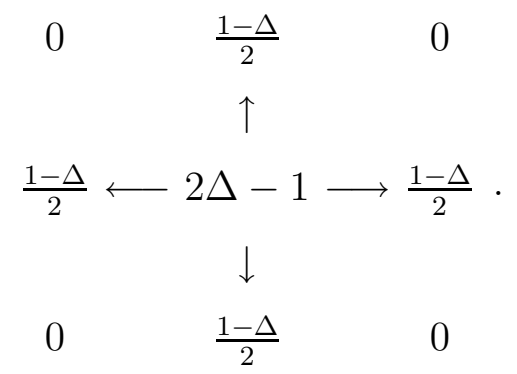

When $\Delta$ is set to 1 we recover the FD stencil while $\Delta=2 / 3$ gives approximately the FE stencil. Choosing the appropriate value of $\Delta$ so as to minimize the error is the subject of the following section. 


\section{Results}

We now test the numerical convergence of the mixed FE-FD scheme by first considering a structure made of perfectly conducting (metallic) cubes of size $\frac{a}{2} \times \frac{a}{2} \times \frac{a}{2}$ embedded in an $\epsilon=\mu=1$ material. The cubes, which form a periodic structure, are separated by distance $a$, the periodicity length. We reproduce here the results of Mias et al. [7] in Section 4.4 of their paper.

Figure 1 (a) shows the second Brillouin zone (the first Brillouin zone which contains the point $\omega=0$ at $\mathbf{k}=0$ is not shown). The solid line was obtained by solving the equation for $\mathbf{E}$ and using 16 bricks along each direction, whereas all other curves were obtained at low $8^{3}$ resolution. The high resolution dispersion curve is in good agreement with the upper band shown in Fig. 12 of Ref. [7]. Due to the variational nature of the FE discretization, it is apparent that the FE method systematically overestimates the eigenfrequency. In contrast, the FD discretization tends to underestimate the eigenfrequency. The mixed FEFD scheme is found to perform best when solving for $\mathbf{H}$. We chose a value $\Delta=5 / 6$, which can be shown to be optimal for plane waves. Despite the departure of the solution from the a plane wave due to scattering occurring at the conductors, the value $\Delta=5 / 6$ can be seen to yield an accuracy that is comparable to the one achieved at higher $16^{3}$ resolution using the standard FE method, an impressive result considering the fact that the $16^{3}$ case takes 50 times longer to run.

Having shown that a single $\Delta$ can improve the accuracy over a wide range of $\mathbf{k}$ values, we now investigate the effect of the mode polarization on the convergence properties by considering a lattice of intersecting conducting rods, which are immersed in an $\epsilon=\mu=1$ medium. Such a geometry has recently attracted interest as a possible means to fabricate media with negative permittivity [8]. Each conductor of cross-section $\frac{a}{3} \times \frac{a}{3}$ is aligned to the principal axes of the $a \times a \times a$ cell. In contrast to the previous test case, such a structure exhibits a cutoff frequency [c.f. Fig. 1 (b)]. In the neighborhood of $\mathbf{k}=0$ (point $\Gamma$ in reciprocal space) two photonic and one plasmon branches emerge with the plasmon eigenvalue $\omega^{2} a^{2} /(2 \pi c)^{2}$ having a weaker dependence in $|\mathbf{k}|^{2}$. At $\mathbf{k} a /(2 \pi)=(0.2,0,0.3)$, however, we found the plasmon to have eigenvalue $\omega^{2} a^{2} /(2 \pi c)^{2}=0.352$, that is only slightly below the two photonic branches 0.386 and 0.408 , respectively. Analysis of the mode structure revealed the plasmon's field to be predominantly aligned to $\mathbf{k}$. In contrast, the two photon fields are oriented mainly perpendicular to $\mathbf{k}$. In spite of the difference in their mode structures, we find in Fig. 2 that the convergence rate can be significantly improved for both types of modes by selecting a single mixture parameter $\Delta$ between 0.8 and 1 .

We now test the capability of Curly3d to handle both positive and negative 

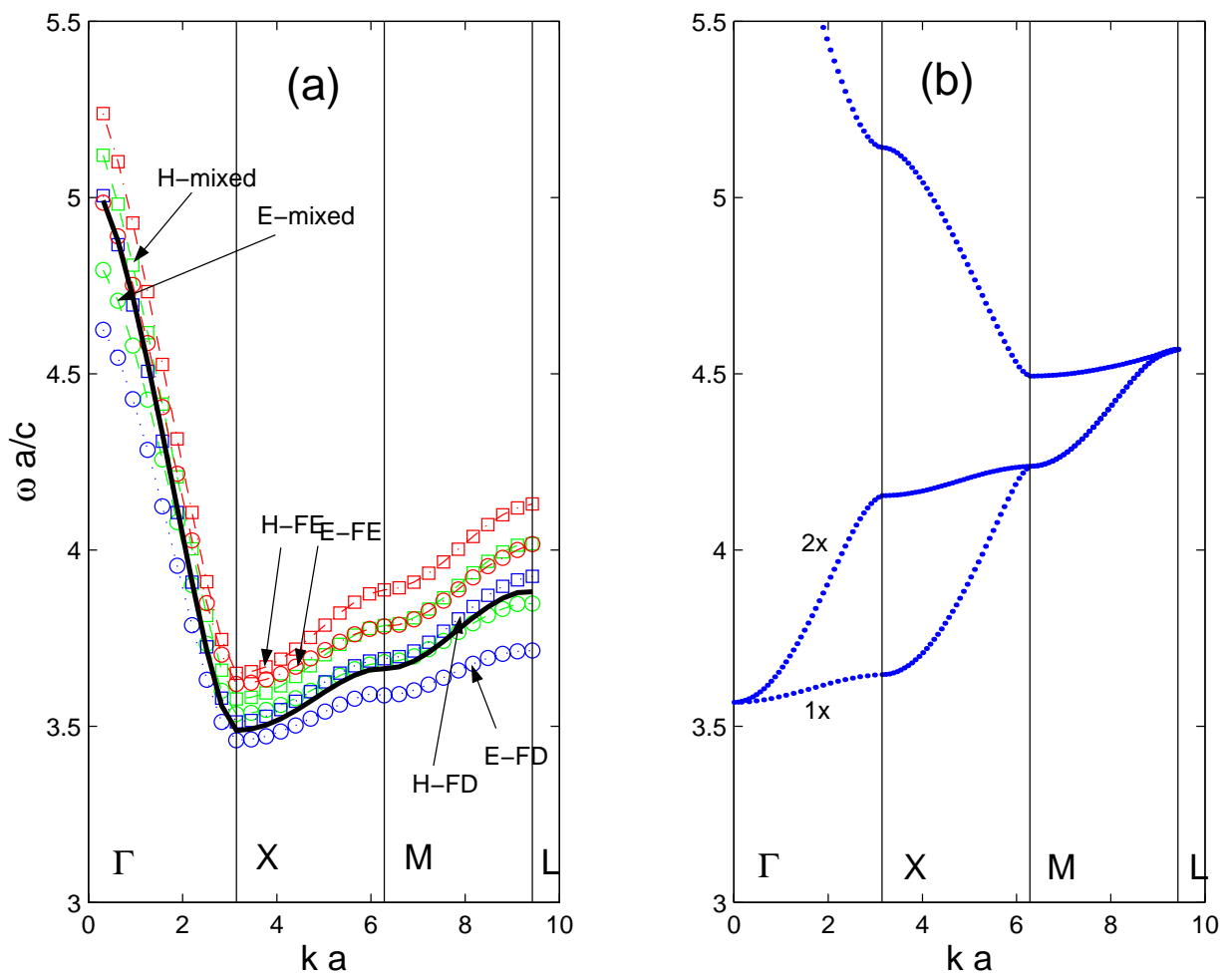

Fig. 1. Dispersion curves obtained by marching in reciprocal space from [000] $\rightarrow$ $[100] \rightarrow[110] \rightarrow[111]$. (a) Metallic cavity of size $a / 2$ : the curves emphasizing the accuracy of the mixed FE-FD scheme were obtained at low $8^{3}$-resolution by solving for $\mathbf{E}$ (circles) and $\mathbf{H}$ (squares) and using three mixture parameters $\Delta$. For reference, the solid curve was obtained using the FE scheme at $16^{3}$-resolution. (b) Dispersion curves for the geometry of intersecting conductors with cutoff at $\omega a / c \approx 3.6$.

$\epsilon$ and $\mu$. Materials with negative $\epsilon$ in a narrow frequency range occur in nature. They include polar crystals $(\mathrm{ZnSe}, \mathrm{SiC})$ and free electron plasmas. The geometry is that of a cubic $\frac{a}{2} \times \frac{a}{2} \times \frac{a}{2}$ insertion of a dielectric with negative permittivity (permeability). Here, we solved for the electric field and kept $\mathbf{k} a /(2 \pi)=(0.5,0,0)$ fixed while decreasing $\epsilon(\mu)$ from 1 to a -1.1 . In the process, the eigensolution at each step was fed to an inverse iteration eigensolver as initial guess to the next step. The eigenfrequency as a function of $\epsilon$ is plotted in Fig. 3. For $\epsilon=\mu=1$, the solution is a plane wave with the numerical eigenfrequency converging to the exact value to within machine accuracy. As $\epsilon$ approaches zero, the electric field becomes increasingly tangential to the dielectric surface and a surface mode develops. The eigenfrequency is ill-defined at $\epsilon=0$. Nonetheless, the mode can be accurately tracked across this point and the eigenfrequency calculation pursued in the negative $\epsilon$ regime. As $\epsilon$ further decreases, sharp features arise at the interface due to the rapid decay of the wave in the negative dielectric, with the normal electric field flipping sign at the surface. This is also a regime where it becomes increasingly difficult to track the mode as the spectrum becomes denser with new modes emerging from below. Varying $\mu$ instead of $\epsilon$ produces a similar curve except for mode 


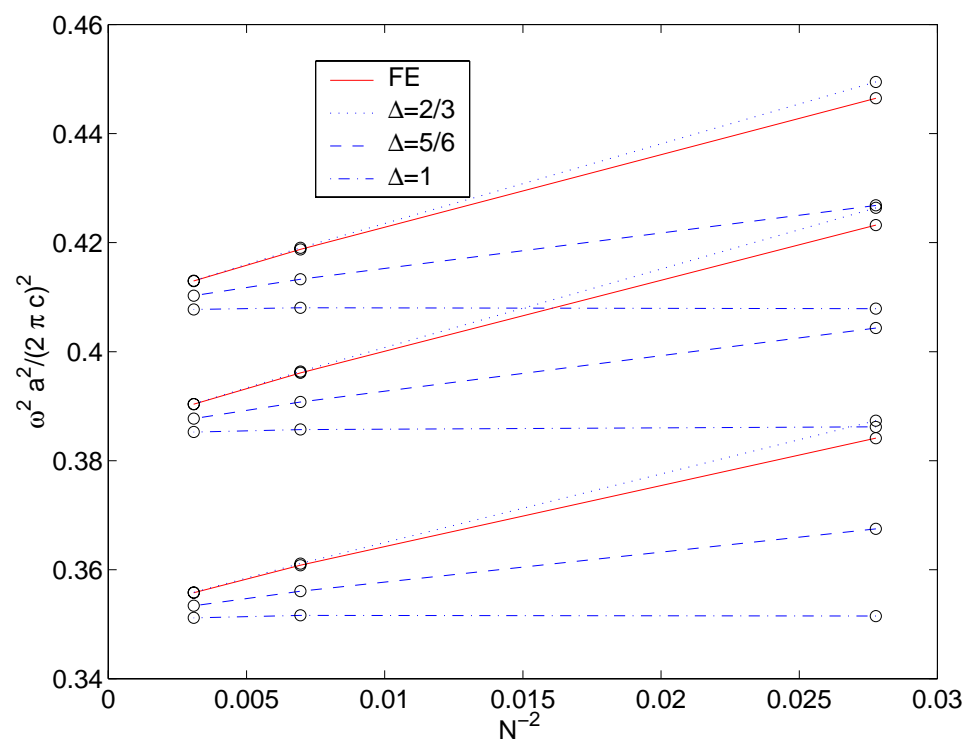

Fig. 2. Convergence of the eigenvalue $\lambda$ in the number $N=N_{x}=N_{y}=N_{z}$ of bricks for the plasmon and photonic modes with conducting rods of size $\frac{a}{3} \times \frac{a}{3}, a=2 \pi$, and $\mathbf{k}=(0.2,0,0.3)$.

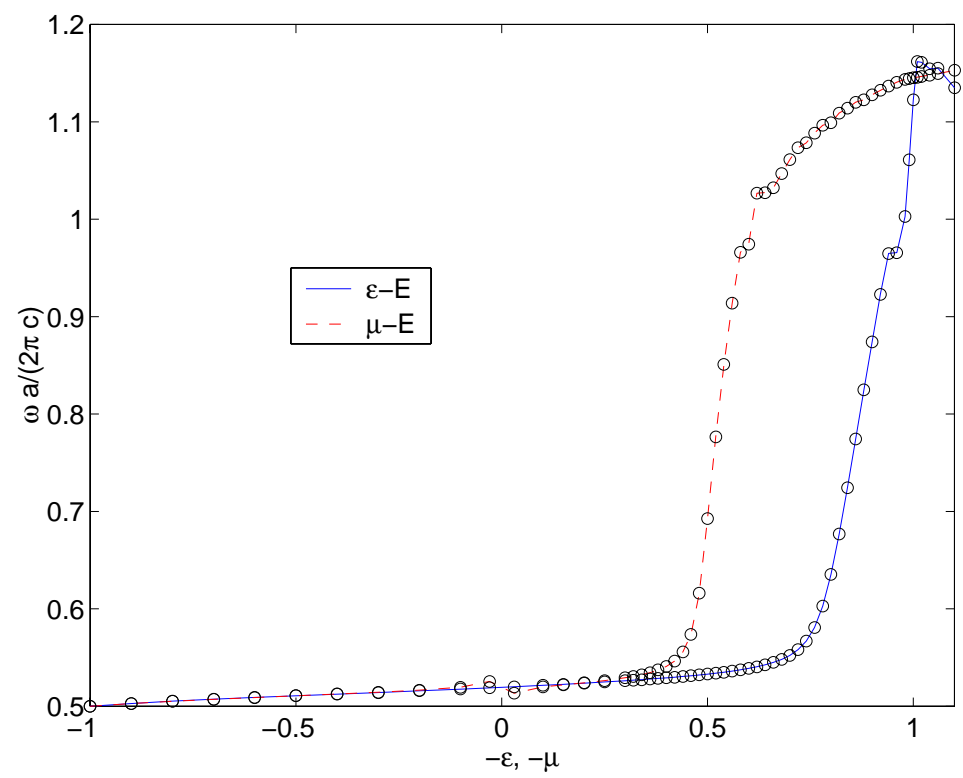

Fig. 3. Dependence of the eigenfrequency on the permittivity (solid line) and permeability (dashed line) inside a cubic of size $\frac{a}{2} \times \frac{a}{2} \times \frac{a}{2}$. Results obtained using $12^{3}$ elements and $\Delta=1$.

conversion occurring in the negative regime. Agreement between the $\epsilon-\mathbf{E}$ and $\mu$-E curves is expected since, by virtue of the duality principle, the equations for $(\mu, \mathbf{E})$ and $(\epsilon, \mathbf{H})$ are indistinguishable. We have tested that individual points on the $\epsilon<-0.4$ branch of the $\epsilon$ - $\mathbf{E}$ curve can also be obtained by running in $\mu-\mathbf{E}$ mode and vice versa so that the appearance of two branches can be explained in terms of coupling to neighboring modes. 


\section{Conclusions}

We have presented a code, Curly3d, designed for the calculations of band structures in metallic and dielectric crystals. Curly3d differs from the MIT Photonic-Band code [9] in that the crystal can have embedded conductors as well as a spatially varying permeability.

Curly3d uses a mixed finite element, finite difference scheme to accelerate the numerical convergence of the eigenvalue calculation. By appropriately tuning the mixing parameter $\Delta$ we were able to show that the accuracy of the numerical results can be improved so as to enable one to run, typically, at half the resolution required by the finite element method. Moreover, the mixing parameter $\Delta$ does not depend sensitively on the wave-vector or on the mode polarization.

A large part of Curly3d, including the matrix assembly, is written in the scripting language Python thus making it easy for users to extend the code. Extensive use of nested lists, hash tables and other advanced data types were made in order to map data on unstructured meshes, composed in our case of uniform bricks. Curly3d relies on the Ellipt2d package [10]. Both Ellipt2d and Curly3d are freely available and can be downloaded from the CVS repository at http://sourceforge.net.

\section{References}

[1] D. R. Smith, W. J. Padilla, D. C. Vier, S. C. Nemat-Nasser, S. Schultz, Composite medium with simultaneously negative permeability and permittivity, Phys. Rev. Letters 84 (2000) 4184.

[2] J. B. Pendry, Negative refraction makes a perfect lens, Phys. Rev. Lett. 85 (2000) 3966.

[3] J. S. van Welij, Calculation of eddy currents in terms of h on hexahedra, IEEE Transactions on Magnetics 21 (1985) 2239.

[4] P. P. Silvester, R. L. Ferrari, Finite Elements for Electrical Engineers, Cambridge University Press, 1996, Ch. 7, pp. 312-316.

[5] C. W. Crowley, P. P. Silvester, H. Hurwitz, Covariant projection elements for 3d vector field problems, IEEE Transactions on Magnetics MAG-18 (1988) 431.

[6] A. Bondeson, G. Y. Fu, Tunable integration scheme for the finite element method, Comput. Phys. Commun. 66 (1991) 167.

[7] C. Mias, J. P. Webb, R. L. Ferrari, Finite element modelling of electromagnetic waves in doubly and triply periodic structures, IEE Proc.-Optoelectron. 146 (1999) 111. 
[8] J. G. Fleming, S. Y. Lin, El-Kady, R. Biswas, K. M. Ho, All-metallic threedimensional photonic crystals with large infrared bandgap, Nature 417 (2002) 52.

[9] S. G. Johnson, J. D. Joannopoulos, Block-iterative frequency-domain methods for maxwell's equations in a planewave basis, Optics Express 8 (2001) 173.

[10] A. Pletzer, Python \& finite elements, Dr. Dobb's Journal 334 (2002) 36. 


\section{External Distribution}

Plasma Research Laboratory, Australian National University, Australia

Professor I.R. J ones, Flinders University, Australia

Professor J oão Canalle, Instituto de Fisica DEQ/IF - UERJ , Brazil

Mr. Gerson O. Ludwig, Instituto Nacional de Pesquisas, Brazil

Dr. P.H. Sakanaka, Instituto Fisica, Brazil

The Librarian, Culham Laboratory, England

Library, R61, Rutherford Appleton Laboratory, England

Mrs. S.A. Hutchinson, JET Library, England

Professor M.N. Bussac, Ecole Polytechnique, France

Librarian, Max-Planck-Institut für Plasmaphysik, Germany

J olan Moldvai, Reports Library, MTA KFKI-ATKI, Hungary

Dr. P. Kaw, Institute for Plasma Research, India

Ms. P.J . Pathak, Librarian, Insitute for Plasma Research, India

Ms. Clelia De Palo, Associazione EURATOM-ENEA, I taly

Dr. G. Grosso, Instituto di Fisica del Plasma, Italy

Librarian, Naka Fusion Research Establishment, J AERI, J apan

Library, Plasma Physics Laboratory, Kyoto University, J apan

Research Information Center, National Institute for Fusion Science, J apan

Dr. O. Mitarai, Kyushu Tokai University, J apan

Library, Academia Sinica, Institute of Plasma Physics, People's Republic of China

Shih-Tung Tsai, Institute of Physics, Chinese Academy of Sciences, People's Republic of China

Dr. S. Mirnov, TRINITI, Troitsk, Russian Federation, Russia

Dr. V.S. Strelkov, Kurchatov Institute, Russian Federation, Russia

Professor Peter Lukac, Katedra Fyziky Plazmy MFF UK, Mlynska dolina F-2, Komenskeho Univerzita, SK-842 15 Bratislava, Slovakia

Dr. G.S. Lee, Korea Basic Science Institute, South Korea

Mr. Dennis Bruggink, Fusion Library, University of Wisconsin, USA

Institute for Plasma Research, University of Maryland, USA

Librarian, Fusion Energy Division, Oak Ridge National Laboratory, USA

Librarian, Institute of Fusion Studies, University of Texas, USA

Librarian, Magnetic Fusion Program, Lawrence Livermore National Laboratory, USA

Library, General Atomics, USA

Plasma Physics Group, Fusion Energy Research Program, University of California at San Diego, USA

Plasma Physics Library, Columbia University, USA

Alkesh Punjabi, Center for Fusion Research and Training, Hampton University, USA

Dr. W.M. Stacey, Fusion Research Center, Georgia Institute of Technology, USA

Dr. J ohn Willis, U.S. Department of Energy, Office of Fusion Energy Sciences, USA

Mr. Paul H. Wright, Indianapolis, Indiana, USA 
The Princeton Plasma Physics Laboratory is operated by Princeton University under contract with the U.S. Department of Energy.

\author{
Information Services \\ Princeton Plasma Physics Laboratory \\ P.O. Box 451 \\ Princeton, NJ 08543
}

Phone: 609-243-2750

Fax: 609-243-2751

e-mail: pppl_info@pppl.gov

Internet Address: http://www.pppl.gov 